\title{
HOMOLOGICAL CHARACTERIZATIONS OF THE APPROXIMATION PROPERTY FOR BANACH SPACES
}

\author{
by YU. V. SELIVANOV
}

(Received 1 March, 1991)

0. Let $E$ be a Banach space, and let $N(E)$ be the Banach algebra of all nuclear operators on $E$. In this work, we shall study the homological properties of this algebra. Some of these properties turn out to be equivalent to the (Grothendieck) approximation property for $E$. These include:

(i) biprojectivity of $N(E)$;

(ii) biflatness of $N(E)$;

(iii) homological finite-dimensionality of $N(E)$;

(iv) vanishing of the three-dimensional cohomology group, $H^{3}(N(E), N(E))$.

This adds another property to the wide range of concepts in functional analysis and topology that can be characterized in terms of topological homology (see [1, Preface, $\S 6])$ : the approximation property for Banach spaces. This property is discussed in [1], [2], [3], and [4].

Let $A$ be a Banach algebra, not necessarily with an identity, and let $A_{+}$be its unitization. All homological concepts to be used below (the cohomology groups of $A$, the groups "Ext", the homological dimension $\mathrm{dh}_{A} X$ of a (left Banach) $A$-module $X$, the (left) global dimension, $\operatorname{dg} A$, and the cohomological dimension (otherwise called bidimension), $\mathrm{db} A$, of $A$, (bi)projectivity, (bi)flatness and others) are assumed to be known; they are set out in detail in A. Ya. Helemskii's book [1] (see also [5]).

Let $B(E)$ (respectively $K(E)$ ) be the Banach algebra of all continuous (respectively all compact) linear operators on $E$. We recall that the algebra $N(E)$ of all nuclear operators on $E$ consists of those elements $T \in B(E)$ which can be represented as an absolutely convergent series $\sum_{n=1}^{\infty} S_{n}$ of one-dimensional operators, and that

$$
\|T\|_{N(E)}=\inf \left\{\sum_{n=1}^{\infty}\left\|S_{n}\right\|_{B(E)}: T=\sum_{n=1}^{\infty} S_{n}\right\} .
$$

As usual, any subalgebra of $B(E)$ that is a Banach algebra with respect to a certain norm $\|.\| \geq\|.\|_{B(E)}$ will be called an operator Banach algebra on $E$. We recall (see [6, Corollary 3.2]) that, if an operator Banach algebra $A$ contains all finite-rank operators on $E$, then it contains $N(E)$ as a continuously embedded bi-ideal of $A$ and is itself a Banach $N(E)$-bimodule in the natural sense. In particular, we can speak about the $N(E)$ bimodules $B(E), K(E)$, and $N(E)$.

Let $E^{*}$ be the dual space of $E$, and let $A(E)=E \hat{\otimes} E^{*}$ be the tensor algebra generated by the duality $\langle x, f\rangle=f(x)\left(x \in E, f \in E^{*}\right)$ (see [6]), with multiplication given by

$$
\left(x_{1} \otimes f_{1}\right)\left(x_{2} \otimes f_{2}\right)=\left\langle x_{2}, f_{1}\right\rangle x_{1} \otimes f_{2} .
$$

Here $\hat{\otimes}$ denotes the projective tensor product of Banach spaces (see [2]). We recall that there exists a homomorphism of Banach algebras $\tau: A(E) \rightarrow B(E)$ defined by $\tau(x \otimes f)(y)=\langle y, f\rangle x$. The image of this homormorphism is the set of operators that can 
be represented in the form

$$
T y=\sum_{n=1}^{\infty}\left\langle y, f_{n}\right\rangle x_{n},
$$

where $x_{n} \in E, f_{n} \in E^{*}$, and $\sum_{n=1}^{\infty}\left\|f_{n}\right\|\left\|x_{n}\right\|<\infty$, i.e., it coincides with $N(E)$. The corestriction of $\tau$ to $N(E)$ is denoted by $\theta: A(E) \rightarrow N(E)$. It is clear that

$$
\|\theta(a)\|_{N(E)} \leq\|a\|_{A(E)} \quad(a \in A(E)) .
$$

Let $L_{E}=\operatorname{ker} \theta$. Then $L_{E}$ is a closed bi-ideal of $A(E)$, and the operator

$$
\bar{\theta}: A(E) / L_{E} \rightarrow N(E)
$$

generated by $\theta$ is an isometric isomorphism of Banach algebras. It is known (see $[2, I, \S 5$, Proposition 35]) that $L_{E}=0$ if and only if $E$ has the approximation property ( $A P$ for short); in this case the Banach algebras $A(E)$ and $N(E)$ are isometrically isomorphic.

By Lemma 2.2 of [6], the algebra $A(E)$ is always biprojective, and therefore (see [1, Theorem V.2.28]) for $A=A(E)$ (and also for $A=N(E)$ in the case where $E$ has $A P$ ) we have $H^{n}(A, X)=0$ for all $A$-bimodules $X$ and for all $n \geq 3$. In particular, $\operatorname{dg} A \leq 2$ and $\mathrm{dh}_{A} \mathbb{C} \leq 1$, where $\mathbb{C}=A_{+} / A$ is the one-dimensional annihilator $A$-module.

The content of the paper is as follows. The key result of Section 1 (Theorem 1) is that, if $E$ does not have $A P$, then, for $A=N(E)$, we have $\mathrm{dh}_{A} A=\infty$. It follows (Corollaries 1 and 2) and $\operatorname{dg} A=\mathrm{dh}_{A} \mathbb{C}=\infty$, and $A$ is neither biprojective nor biflat. In Corollaries 3 and 4, Theorem 1 is used to study the cohomology groups $H^{n}(A, X)$ and singular extensions of the algebra $A=N(E)$ by $X$, where $X$ is a right-annihilator $A$-bimodule. In Section 2, the global and cohomological dimensions (and other characteristics) of the algebras $A(E)$ and $N(E)$ are calculated for each infinitedimensional Banach space $E$. We show (Theorem 2 and Corollary 5) that $\operatorname{dg} A(E)=$ $\mathrm{db} A(E)=2$, and the condition that $\operatorname{dg} N(E)=2(\mathrm{db} N(E)=2)$ is equivalent to the approximation property for $E$. Section 3 studies the cohomology groups of the algebra $N(E)$ with coefficients in annihilator and some other bimodules. In particular, Theorem 6 shows that $H^{2}(N(E), N(E))=0$ for each Banach space $E$, and that $H^{3}(N(E), N(E))=0$ if and only if $E$ has $A P$.

1. Homological finite-dimensionality for the algebra of nuclear operators. We shall prove the following theorem.

Theorem 1. Let $E$ be a Banach space, and let $A=N(E)$. Then

$$
\mathrm{dh}_{A} A= \begin{cases}0 & \text { if } E \text { has } A P, \\ \infty & \text { if } E \text { does not have } A P .\end{cases}
$$

We preface to the proof of Theorem 1 a number of simple lemmas.

Lemma 1. Let $E$ be a Banach space. Then, for each $a, b \in A(E)=E \hat{\otimes} E^{*}$ and each $L \in B(E)$, the following equalities hold:

(i) $a b=\left(\tau(a) \hat{\otimes} 1_{E^{*}}\right)(b)=\left(1_{E} \hat{\otimes}(\tau(b))^{*}\right)(a)$;

(ii) $\tau(a) L=\tau\left(\left(1_{E} \hat{\otimes} L^{*}\right)(a)\right.$ and $L \tau(a)=\tau\left(\left(L \hat{\otimes} 1_{E^{*}}\right)(a)\right)$. 
If , in addition, $x_{1} \otimes f_{1}, x_{2} \otimes f_{2} \in A(E)$, then we have:

(iii) $a\left(x_{1} \otimes f_{1}\right)=\tau(a)\left(x_{1}\right) \otimes f_{1}$ and $\left(x_{1} \otimes f_{1}\right) a=x_{1} \otimes(\tau(a))^{*}\left(f_{1}\right)$;

(iv) $\left(x_{1} \otimes f_{1}\right) a\left(x_{2} \otimes f_{2}\right)=\left\langle\tau(a)\left(x_{2}\right), f_{1}\right\rangle x_{1} \otimes f_{2}$;

(v) $a\left(x_{1} \otimes f_{1}\right) b=\tau(a)\left(x_{1}\right) \otimes(\tau(b))^{*}\left(f_{1}\right)$.

Proof. Formulae (ii) were established in [6] (see the proof of Theorem 3.2). The equalities (i) and (iii) are easy to check on elementary tensors. The formulae (iv) and (v) follows from (iii).

For an algebra $A$, we set

$$
\begin{aligned}
& \operatorname{Lan}(A)=\{a \in A: a b=0 \text { for all } b \in A\}, \\
& \operatorname{Ann}(A)=\{a \in A: a b=b a=0 \text { for all } b \in A\} .
\end{aligned}
$$

Lemma 2. Let $E$ be a Banach space, and let $A=A(E)$. Then $L_{E}=\operatorname{Ann}(A)=\operatorname{Lan}(A)$.

Proof. We recall that $L_{E}=\operatorname{Ker} \theta=\operatorname{Ker} \tau$. Let $a \in L_{E}$. Then $\tau(a)=0$ and, by Lemma 1(i), $a b=b a=0$ for $b \in A$. Hence $a \in \operatorname{Ann}(A)$. Thus $L_{E} \subset \operatorname{Ann}(A)$. Since always $\operatorname{Ann}(A) \subset \operatorname{Lan}(A)$, it remains to prove that $\operatorname{Lan}(A) \subset L_{E}$.

Let $a \in \operatorname{Lan}(A)$. Then for $b=x \otimes f \in A(E)$ we have $a b=0$, where $a b=\tau(a)(x) \otimes f$, by Lemma 1(iii). Since $x \in E$ and $f \in E^{*}$ are arbitrary, we find that $\tau(a)=0$ i.e., $a \in \operatorname{Ker} \tau=L_{E}$.

It is clear that every Banach space $E$ is a left Banach $N(E)$-module provided that the outer multiplication is defined as the action of the operator on the vector. The space $E^{*}$ is now regarded as the right Banach $N(E)$-module dual to $E$. Let $E \hat{\otimes} E^{*}$ be the $N(E)$-bimodule obtained from $E$ and $E^{*}$ by the tensor product bifunctor (see [1, II, \$5.3]). It is obvious that the outer multiplications of $E \hat{\otimes} E^{*}$ by elements of $N(E)$ are given by the formulae

$$
L . u=\left(L \hat{\otimes} 1_{E^{*}}\right)(u), \quad \text { u. } L=\left(1_{E} \hat{\otimes} L^{*}\right)(u),
$$

where $L \in N(E)$ and $u \in E \hat{\otimes} E^{*}$. By Lemma 1(ii), the operator $\theta: E \hat{\otimes} E^{*} \rightarrow N(E)$ is a morphism of $N(E)$-bimodules.

We now recall (see [1, II, §5.3]) that there is the so-called reduced module $X_{\Pi}=A \hat{\otimes}_{A} X$ associated with any left $A$-module $X$. Let $\mathrm{K}: X_{\Pi} \rightarrow X$ be the morphism of $A$-modules defined by $\kappa(a \underset{A}{\otimes} x)=a . x$. If $X=A$, then it is easy to turn $X_{\mathrm{n}}=A \hat{\bigotimes}_{A} A$ into $A$-bimodule (see [1, Proposition II.5.15]). In this case, $\kappa: A \underset{A}{\hat{\otimes}} A \rightarrow A$ becomes a morphism of $A$-bimodules.

Lemma 3 (see [7, 4.6(i)]). Let $E$ be a Banach space, and let $A=N(E)$. Then, up to an isometric isomorphism of $A$-bimodules, the reduced module $A_{\Pi}=A \hat{\otimes} A$ coincides with $E \hat{\otimes} E^{*}$, and the morphism $\kappa: A_{\Pi} \rightarrow A$ coincides with the epimorphism $\theta: E \hat{\otimes} E^{*} \rightarrow$ $N(E)$.

We give a direct proof of this result.

Proof. Choose $x_{0} \in E$ and $f_{0} \in E^{*}$ such that $\left\langle x_{0}, f_{0}\right\rangle=\left\|x_{0}\right\|_{E}=\left\|f_{0}\right\|_{E^{*}}=1$. For $x \in E$, $f \in E^{*}$, let

$$
\lambda(x \otimes f)=\theta(x \otimes f) \underset{A}{\otimes} \theta\left(x_{0} \otimes f\right) .
$$




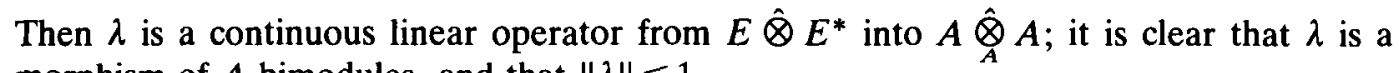
morphism of $A$-bimodules, and that $\|\lambda\| \leq 1$.

On the other hand, let $R: A \times A \rightarrow E \hat{\otimes} E^{*}$ be the bilinear operator given by $R(\theta u, \theta v)=u v$, where $u, v \in A(E)$. We shall prove that $R$ is properly defined and continuous. Indeed, if $\theta u^{\prime}=\theta u$ and $\theta v^{\prime}=\theta v$, then, by Lemma $2, u^{\prime}-u, v^{\prime}-v \epsilon$ $\operatorname{Ker} \theta=L_{E}=\operatorname{Ann}(A(E))$; hence $u^{\prime} v^{\prime}=u v$ and

$$
\|u v\| \leq\left\|u+L_{E}\right\|\left\|v+L_{E}\right\|=\|\theta u\|\|\theta v\| .
$$

It is easily verified that $R$ is balanced (i.e., $R(a b, c)=R(a, b c)$ for any $a=\theta u$, $b=\theta v, c=\theta w$, where $u, v, w \in A(E))$. The operator from $A \hat{\otimes} A$ into $E \hat{\otimes} E^{*}$ associated with $R$ is denoted by $\mu$. It is obvious that $\mu$ is a morphism of $A$-bimodules, that $\|\mu\| \leq 1$, and that $\mu \circ \lambda=1_{E \dot{\otimes} E^{*}}$. We shall prove now that $\lambda \circ \mu$ is the identity on $A \hat{\otimes}_{A} A$, in which case $\lambda=\mu^{-1}$ and $\mu: A \underset{A}{\otimes} A \rightarrow E \hat{\otimes} E^{*}$ is an isometric isomorphism of $A$ bimodules.

Indeed, since $\theta: A(E) \rightarrow N(E)$ is a homomorphism of algebras, we have

$$
\begin{aligned}
& (\lambda \circ \mu)(\theta u \underset{A}{\otimes} \theta v)=\lambda(u v) \\
& =\left\langle x_{2}, f_{1}\right\rangle \lambda\left(x_{1} \otimes f_{2}\right) \\
& =\left\langle x_{2}, f_{1}\right\rangle \theta\left(x_{1} \otimes f_{0}\right) \otimes \theta \theta\left(x_{0} \otimes f_{2}\right) \\
& =\theta\left(x_{1} \otimes f_{0}\right){ }_{A} \theta\left(\left(x_{0} \otimes f_{1}\right) v\right) \\
& =\theta\left(x_{1} \otimes f_{0}\right){ }_{A} \theta\left(x_{0} \otimes f_{1}\right) \theta v \\
& =\theta\left(x_{1} \otimes f_{0}\right) \theta\left(x_{0} \otimes f_{1}\right) \otimes \theta \\
& =\theta u \otimes \theta v
\end{aligned}
$$

for arbitrary elementary tensors $u=x_{1} \otimes f_{1}$ and $v=x_{2} \otimes f_{2}$.

It remains only to note that $k=\theta \circ \mu$, and the assertion is proved.

Lemma 4. Let $E$ be a Banach space, and set $A=N(E)$. Then the left $A$-module $A_{\Pi}$ is projective and the following statements are equivalent:

(i) the left A-module $A$ is projective;

(ii) the left A-module $A$ is flat;

(iii) $E$ has $A P$.

Proof. Let $x_{0} \in E$ and $f_{0} \in E^{*}$ be such that $\left\langle x_{0}, f_{0}\right\rangle=1$. It is easy to see that the formula

$$
\rho(x \otimes f)=\theta\left(x \otimes f_{0}\right) \otimes\left(x_{0} \otimes f\right) \quad\left(x \in E, f \in E^{*}\right)
$$

defines a morphism of left Banach $A$-modules

$$
\rho: E \hat{\otimes} E^{*} \rightarrow A \hat{\otimes}\left(E \hat{\otimes} E^{*}\right) \subset A_{+} \hat{\otimes}\left(E \hat{\otimes} E^{*}\right)
$$


such that $\pi \circ \rho$ is the identity on $E \hat{\otimes} E^{*}$, where $\pi: A \hat{\otimes}\left(E \hat{\otimes} E^{*}\right) \rightarrow E \hat{\otimes} E^{*}$ (the so-called canonical morphism) is defined by $\pi(a \otimes u)=a . u\left(a \in A, u \in E \hat{\otimes} E^{*}\right)$. It follows that the $A$-module $E \hat{\otimes} E^{*}$ is projective. Since, by Lemma 3 , the $A$-module $A_{\Pi}$ is isomorphic to $E \hat{\otimes} E^{*}, A_{\Pi}$ is also projective.

The implication (i) $\Rightarrow$ (ii) follows from [5, Proposition 7.1.44].

Now suppose that (ii) holds. Let $i$ be the natural embedding of $A$ in $A_{+}$. By [5, Theorem 7.1.42], the operator $K=i \hat{\otimes} 1_{A}: A \hat{\otimes} A \rightarrow A_{+} \hat{\otimes}_{A} A=A$ is topologically injective. In particular, $\operatorname{ker} K=0$. By Lemma 3 , $\operatorname{ker} \theta=0$ and, consequently, (iii) holds.

The implication (iii) $\Rightarrow$ (i) follows from the facts that, for $E$ having $A P, \theta: E \hat{\otimes} E^{*} \rightarrow$ $A$ is an isomorphism of left $A$-modules, and $E \hat{\otimes} E^{*}$ is always projective.

Proof of Theorem 1 . The case " $E$ has $A P$ " is contained in Lemma 4.

We now assume that $E$ does not have $A P$. Set $n=\mathrm{dh}_{A} A$, and suppose that $n<\infty$. By Lemma $4, n \geq 1$. By Theorem [1, V.2.1], for some $A$-module $W$ there exist short admissible complexes of $A$-modules

and

$$
0 \leftarrow A \leftarrow U \leftarrow W \leftarrow 0
$$

$$
0 \leftarrow W \leftarrow V \leftarrow A \hat{\otimes} A_{\Pi} \leftarrow 0,
$$

where $U=\left(A_{+} \hat{\otimes} A\right) \otimes A_{\Pi}, V=\left(A_{+} \hat{\otimes} A_{\pi}\right) \oplus(A \hat{\otimes} A)$,

$$
\Delta(a \otimes x)=(i(a) \otimes x, a \otimes \kappa(x)) \quad\left(a \in A, x \in A_{\Pi}\right),
$$

$i$ is the natural embedding of $A$ in $A_{+}$, and $\kappa=i \underset{A}{\hat{\otimes}} 1_{A}: A_{\Pi} \rightarrow A$. By Lemma $4, \mathrm{dh}_{A} U=0$ and, by [1, Proposition III.5.5],

$$
\mathrm{dh}_{A} W \leq \max \left\{\mathrm{dh}_{A} U, \mathrm{dh}_{A} A-1\right\} \leq n-1 .
$$

The short admissible complex (1) defines, for any $A$-module $Y$, an exact sequence of groups

$$
\ldots \rightarrow \operatorname{Ext}_{A}^{n}(W, Y) \rightarrow \operatorname{Ext}_{A}^{n}(V, Y) \rightarrow \operatorname{Ext}_{A}^{n}\left(A \hat{\otimes} A_{\Pi}, Y\right) \rightarrow \operatorname{Ext}_{A}^{n+1}(W, Y) \rightarrow \ldots
$$

(see [1, Theorem III.4.4]). Since $\operatorname{dh}_{A} W \leq n-1$, we have $\operatorname{Ext}_{A}^{n}(W, Y)=\operatorname{Ext}_{A}^{n+1}(W, Y)=$ 0 . Since the $A$-module $A_{+} \hat{\otimes} A_{\Pi}$ is projective, it follows that $\operatorname{Ext}_{A}^{n}(V, Y)=$ $\operatorname{Ext}_{A}^{n}(A \hat{\otimes} A, Y)$, recalling that $n \geq 1$. Consequently, the segment (2) of the long exact sequence for the groups Ext takes the form

$$
0 \rightarrow \operatorname{Ext}_{A}^{n}(A \hat{\otimes} A, Y) \stackrel{s}{\rightarrow} \operatorname{Ext}_{A}^{n}\left(A \hat{\otimes} A_{\Pi}, Y\right) \rightarrow 0 .
$$

Thus the morphism of groups $\delta=\operatorname{Ext}_{A}^{n}\left(1_{A} \hat{\otimes} \kappa, Y\right)$ is an isomorphism for any $A$-module $Y$.

Let

$$
0 \leftarrow A \stackrel{\varepsilon}{\longleftarrow} P_{0} \stackrel{d_{0}}{\longleftarrow} \ldots \stackrel{d_{n-2}}{\longleftarrow} P_{n-1} \stackrel{d_{n-1}}{\longleftarrow} P_{n} \leftarrow 0
$$

be a projective resolution of length $n$ for $A$-module $A$. (Such a resolution exists because 
$\mathrm{dh}_{A} A=n$.) Consider the commutative diagram

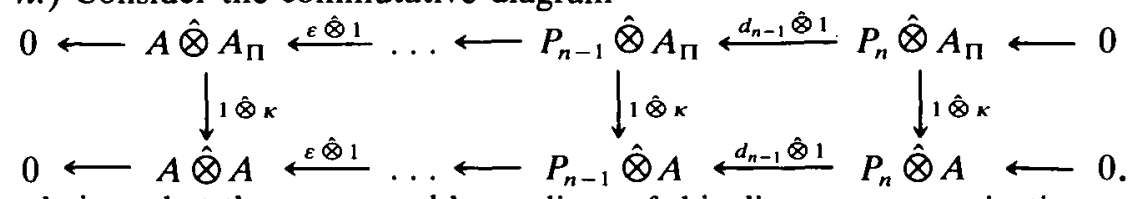

It is obvious that the upper and lower lines of this diagram are projective resolutions of the $A$-modules $A \hat{\otimes} A_{\Pi}$ and $A \hat{\otimes} A$, respectively.

Let $Y$ be an arbitrary $A$-module. Applying the functor ${ }_{A} h(?, Y)$ to the diagram (3), we obtain the commutative diagram

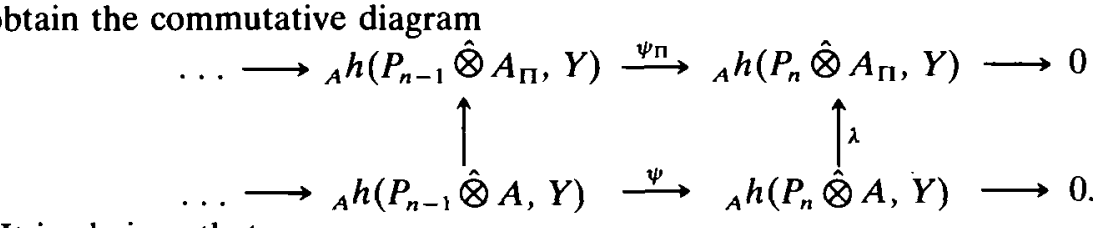

It is obvious that

and

$$
\operatorname{Ext}_{A}^{n}\left(A \hat{\otimes} A_{\Pi}, Y\right)={ }_{A} h\left(P_{n} \hat{\otimes} A_{\Pi}, Y\right) / \operatorname{Im} \psi_{\mathrm{n}}
$$

$$
\operatorname{Ext}_{A}^{n}(A \hat{\otimes} A, Y)={ }_{A} h\left(P_{n} \hat{\otimes} A, Y\right) / \operatorname{Im} \psi,
$$

and that the operator $\lambda={ }_{A} h(1 \hat{\otimes} \kappa, Y)$ generates the morphism $\delta$ considered above. As was shown, $\delta$ is an isomorphism.

Now set $Y=P_{n} \hat{\otimes} A_{\Pi}$, and consider the element of the group $\operatorname{Ext}_{A}^{n}\left(A \hat{\otimes} A_{\Pi}, Y\right)=$ ${ }_{A} h(Y, Y) / \operatorname{Im} \psi_{\Pi}$ defined by $1_{Y} \in{ }_{A} h(Y, Y)$. This element belongs to $\operatorname{Im} \delta$. It follows that there exist morphisms of $A$-modules $\xi: P_{n-1} \hat{\otimes} A_{\Pi} \rightarrow Y$ and $\eta: P_{n} \hat{\otimes} A \rightarrow Y$ such that

$$
1_{Y}=\psi_{\Pi}(\xi)+\lambda(\eta)
$$

But $\psi_{\Pi}(\xi)=\xi \circ\left(d_{n-1} \hat{\otimes} 1\right)$ and $\lambda(\eta)=\eta \circ\left(1 \hat{\otimes}_{\kappa}\right)$, and hence

$$
x \otimes y=\xi\left(d_{n-1}(x) \otimes y\right)+\eta(x \otimes \kappa(y)) \quad\left(x \in P_{n}, y \in A_{\Pi}\right) .
$$

Since $E$ does not have $A P$, by Lemma 3, ker $K \neq 0$. Let $y_{0} \in \operatorname{ker} \kappa \backslash\{0\}$ and $f_{0} \in\left(A_{\Pi}\right)^{*}$ be such that $f_{0}\left(y_{0}\right)=1$. Set

$$
\zeta(z)=\left(1 \hat{\otimes} f_{0}\right) \xi\left(z \otimes y_{0}\right) \quad\left(z \in P_{n-1}\right) .
$$

Then clearly $\zeta: P_{n-1} \rightarrow P_{n}$ is a morphism of $A$-modules. From (4) for $y=y_{0}$, we see that

$$
x=\left(1 \otimes f_{0}\right)\left(x \otimes y_{0}\right)=\zeta\left(d_{n-1}(x)\right)
$$

for all $x \in P_{n}$, and hence $\zeta \circ d_{n-1}$ is the identity on $P_{n}$. Consequently the morphism $d_{n-1}: P_{n} \rightarrow P_{n-1}$ is a coretraction. But then obviously $\mathrm{dh}_{A} A<n$. Since $n=\mathrm{dh}_{A} A$, we obtain a contradiction. Thus $\mathrm{dh}_{A} A=\infty$, and the theorem is proved.

The following corollary is a consequence of Theorem 1, [1, Theorem V.2.28] and [6, Lemma 2.2].

Corollary 1. Let $E$ be a Banach space, and let $A=N(E)$. Then the following statements are equivalent:

(i) $A$ is biprojective;

(ii) $H^{3}(A, X)=0$ for all $A$-bimodules $X$;

(iii) $\operatorname{dg} A<\infty$;

(iv) $\mathrm{dh}_{A} \mathbb{C}<\infty$;

(v) $E$ has $A P$. 
We recall (see [1, Proposition VII.2.2]) that, if $A$ is a biflat Banach algebra, then the left $A$-module $A$ is flat. On the other hand, every biprojective Banach algebra is biflat. From Lemma 4 and Corollary 1 we obtain the following corollary.

Corollary 2. Let $E$ be a Banach space. The Banach algebra $N(E)$ is biflat if and only if $E$ has $A P$.

We recall that an $A$-bimodule $X$ is called right-annihilator if $x . a=0$ for all $x \in X$, $a \in A$. Each right-annihilator $A$-bimodule $X$ can be regarded as the $A$-bimodule $B(\mathbb{C}, X)$ (see [1, Proposition 0.4.5]). Theorem 1 and the formula

$$
H^{n}(A, B(\mathbb{C}, X))=\operatorname{Ext}_{A}^{n}(\mathbb{C}, X)
$$

(see [1, Theorem III.4.12]) yield the following corollary.

Corollary 3. Let $E$ be a Banach space, and let $A=N(E)$. all $n \geq 2$.

(i) If $E$ has $A P$, then $H^{n}(A, X)=0$ for all right-annihilator $A$-bimodules $X$ and for

(ii) If $E$ does not have $A P$, then, for each $n$, there exists a right-annihilator $A$ bimodule $X$ such that $H^{n}(A, X) \neq 0$.

As is well known (see $[1, I, \S 1.2]$ ), the question of the triviality of two-dimensional cohomology groups of a Banach algebra $A$ with coefficients in $A$-bimodules is closely connected with the question of the splitting of singular extensions of this algebra. In particular, we obtain the next corollary from Corollary 3 and [1, Corollary I.1.11].

Corollary 4. Let $E$ be a Banach space, and let $A=N(E)$.

(i) If $E$ has $A P$, then any singular extension of the algebra $A$ by a right-annihilator A-bimodule splits.

(ii) If $E$ does not have $A P$, then there exists an unsplittable singular extension of the algebra $A$ by a right-annihilator A-bimodule.

2. The global dimension of the algebra of nuclear operators. The first theorem is related to $[8$, Theorem 5], which was stated without proof.

THEOREM 2. Let $E$ be an infinite-dimensional Banach space, and let $A=A(E)$. Then $\mathrm{dh}_{A} K(E)=\mathrm{dh}_{A} B(E)=2$, and $\operatorname{dg} A=\mathrm{db} A=2$.

To prove Theorem 2, we need a lemma.

Lemma 5. Let $E$ be a Banach space, and set $A=A(E)$. Suppose also that $X=K(E)$ or $B(E)$. Then, up to an isometric isomorphism of $A$-bimodules, the reduced modules $A \underset{A}{\otimes} X$ and $X \underset{A}{\hat{Q}} A$ coincide with $E \hat{\otimes} E^{*}$.

The proof of this lemma is analogous to the proof of Lemma 3 ; the isomorphism $\mu: A \underset{A}{\otimes} X \rightarrow E \hat{\otimes} E^{*}$ is defined by

$$
\mu((x \otimes f) \underset{A}{\otimes} L)=x \otimes L^{*} f \quad\left(x \in E, L \in X, f \in E^{*}\right)
$$


Proof of Theorem 2. Let $X=K(E)$ or $B(E)$. By [1, Theorem V.2.1], there exists a resolution for the $A$-module $X$ of the form

$$
0 \leftarrow X \leftarrow U \leftarrow V \leftarrow A \hat{\otimes} X_{\mathbf{n}} \leftarrow 0,
$$

where $U=\left(A_{+} \hat{\otimes} X\right) \oplus X_{\Pi}, V=\left(A_{+} \hat{\otimes} X_{\Pi}\right) \oplus(A \hat{\otimes} X)$,

$$
\Delta(a \otimes x)=(i(a) \otimes x, a \otimes \kappa(x)) \quad\left(a \in A, x \in X_{\mathrm{n}}\right),
$$

$i$ is the natural embedding of $A$ in $A_{+}$, and $\kappa=i \hat{\otimes}_{A} 1_{X}: X_{\Pi}=A \underset{A}{\hat{\otimes}} X \rightarrow X$. By Lemma 5 , $X_{\Pi}=A$ up to an isomorphism. Since the algebra $A$ is projective, it is clear that the complex (5) is a projective resolution. Its length is equal to 2 . To obtain a contradiction, suppose that $\mathrm{dh}_{A} X<2$. Then obviously the morphism $\Delta$ is a coretraction, i.e., there exists a morphism of $A$-modules $\nabla: V \rightarrow A \hat{\otimes} X_{\mathrm{n}}$ such that $\nabla \circ \Delta$ is the identity on $A \hat{\otimes} X_{\Pi}$. Set $\Delta_{1}=1_{X} \hat{\otimes} \Delta$ and $\nabla_{1}=1_{X} \hat{\otimes} \nabla$. Then, by Lemma 5 ,

and

$$
\Delta_{1}: A \hat{\otimes} A \rightarrow(X \hat{\otimes} A) \oplus(A \hat{\otimes} X)
$$

$$
\Delta_{1}(a \otimes b)=(\tau(a) \otimes b, a \otimes \tau(b)) \quad(a, b \in A) .
$$

Since obviously $\nabla_{1} \circ \Delta_{1}$ is the identity on $A \hat{\otimes} A$, we have for each $a \in A$,

$$
\begin{aligned}
\|a\|^{2} & =\|a \otimes a\|=\left\|\nabla_{1}\left(\Delta_{1}(a \otimes a)\right)\right\| \\
& \leq C(\|\tau(a) \otimes a\|+\|a \otimes \tau(a)\|) \\
& \leq 2 C\|a\|\|\tau(a)\|_{X},
\end{aligned}
$$

i.e., $\|a\| \leq 2 C\|\tau(a)\|_{X}$, where $C=\left\|\nabla_{1}\right\|$. It follows that $L_{E}=\operatorname{ker} \tau=0$ and that the norms of the spaces $E \hat{\otimes} E^{*}=A$ and $E E \subset X$ are equivalent. (Here, $\otimes$ is the symbol for the weak tensor product of Banach spaces (see [2], [5]).) In other words, $E \hat{\otimes} E^{*}$ and $E \otimes E^{*}$ are canonically isomorphic. As Grothendieck showed (see $[2, \mathrm{I}, \S 4$, Corollary 2 on p. 153]), the latter implies that $E$ is finite-dimensional. But we have assumed that $\operatorname{dim} E=\infty$, and so we have a contradiction.

Consequently, $\operatorname{dh}_{A} X=2$ and $\mathrm{db} A \geq \operatorname{dg} A \geq 2$. Since the algebra $A$ is biprojective ([6, Lemma 2.2]), [1, Theorem V.2.28] implies that $\mathrm{db} A \leq 2$. Hence $\operatorname{dg} A=\mathrm{db} A=2$. The proof is complete.

Corollary 1 and Theorem 2 yield the following corollary. Then

Corollary 5. Let $E$ be an infinite-dimensional Banach space, and let $A=N(E)$.

$$
\operatorname{dg} A=\mathrm{db} A= \begin{cases}2 & \text { if } E \text { has } A P, \\ \infty & \text { if } E \text { does not have } A P .\end{cases}
$$

The following corollary is a consequence of Lemmas 3 and 5, [1, Proposition II.3.13] and Grothendieck's results which was used in the proof of Theorem 2.

Corollary 6. Let $E$ be a Banach space, and let $A=A(E)$ or $N(E)$. Then $A$ has a left bounded approximate identity if and only if $\operatorname{dim} E<\infty$. 
TheOrem 3. Let $E$ be a Banach space, and let $A=A(E)$ and $N=N(E)$. Then

$$
\mathrm{dh}_{A} N= \begin{cases}0 & \text { if } E \text { has } A P, \\ 2 & \text { if } E \text { does not have } A P .\end{cases}
$$

The proof of this theorem is analogous to the proof of Theorem 2 .

3. The cohomologies of the algebra of nuclear operators with special coefficients. We recall that an $A$-bimodule $X$ is called annihilator if $a . x=x . a=0$ for all $a \in A, x \in X$.

Theorem 4. Let $E$ be a Banach space, and set $N=N(E)$. Let $X \neq 0$ be an annihilator A-bimodule. Then:

(i) $H^{2}(N, X)=0 \quad$ if and only if $E$ has $A P$;

(ii) $H^{3}(N, X)=0$.

Proof. The assertion (i) follows at once from [1, Theorem II.3.23] and Lemma 3.

Now let $f \in Z^{3}(N, X)$, i.e., let $f$ be a continuous trilinear operator from $N \times N \times N$ to $X$ such that the identity

$$
f(a, b c, d)=f(a b, c, d)+f(a, b, c d) \quad(a, b, c, d \in N)
$$

holds. Set $A=A(E)$ and set

$$
f_{1}(a, b, c)=f(\theta a, \theta b, \theta c) \quad(a, b, c \in A) .
$$

Then $f_{1}$ is a continuous trilinear operator from $A \times A \times A$ to $X$. Since $\theta: A \rightarrow N$ is a homomorphism of Banach algebras, it is obvious that $f_{1} \in Z^{3}(A, X)$. It follows from the biprojectivity of the algebra $A$ that $H^{3}(A, X)=0$. Hence $f_{1}=\delta^{2} g_{1}$ for some $g_{1}: A \times A \rightarrow$ $X$ which is a continuous bilinear operator, i.e., we have

$$
f_{1}(a, b, c)=-g_{1}(a b, c)+g_{1}(a, b c) \quad(a, b, c \in A) .
$$

If $a \in L_{E}=\operatorname{ker} \theta$, then for any $b, c \in A$ we have $f_{1}(a, b, c)=0$, and, by Lemma 2, $g_{1}(a b, c)=0$. Hence $g_{1}(a, b c)=f_{1}(a, b, c)+g_{1}(a b, c)=0$. Consequently, $g_{1}\left(L_{E}, A\right)=0$. It is similarly shown that $g_{1}\left(A, L_{E}\right)=0$.

It is obvious that the formula

$$
g(\theta a, \theta b)=g_{1}(a, b) \quad(a, b \in A)
$$

defines a continuous bilinear operator $g: N \times N \rightarrow X$ such that $f=\delta^{2} g$. This completes the proof of (ii).

It follows from Theorem 4 that, if a Banach space $E$ does not have $A P$, then, for any annihilator $N(E)$-bimodule $X \neq 0$, there exists an unsplittable singular extension of $N(E)$ by $X$. On the other hand, if $E$ has $A P$, then one can show the following: any extension of $N(E)$ by an annihilator $N(E)$-bimodule splits, even if it is not a priori singular.

Now let $E$ be a Banach space, and let $j$ be the natural embedding of $L_{E}=\operatorname{ker} \theta$ in $A(E)$. Since

$$
0 \rightarrow L_{E} \stackrel{\dot{\leftrightarrow}}{\rightarrow} A(E) \stackrel{\theta}{\rightarrow} N(E) \rightarrow 0
$$

is an exact sequence of Banach algebras and $L_{E}=\operatorname{Ann}(A(E))$, the triple $(A(E), \theta, j)$ is an extension of the algebra $N(E)$ by the annihilator $N(E)$-bimodule $L_{E}$. It is not clear to 
the author whether this extension is singular (i.e., whether $L_{E}$ has, as a subspace, a Banach complement in $A(E)$ for any $E$ ). But one can show that whether or not it splits is equivalent to the approximation property for $E$.

We continue to study the cohomology groups of the algebra $N(E)$. The proof of the first lemma is analogous to the proof of $[9$, Theorem 1].

Lemma 6. Let $E$ be a Banach space, and let $A$ be $N(E)$ or $A(E)$. Then $H^{n}(A, B(E))=0$ for all $n \geq 1$.

Theorem 5. Let $E$ be a Banach space, and let $A$ be $N(E)$ or $A(E)$. Let $B$ be an operator Banach algebra on $E$ containing all finite-rank operators on $E$. Then $B$ is a Banach A-bimodule and $H^{2}(A, B)=0$.

Proof. According to [6, Corollary 3.2], $N(E) \subset B$ and the embedding of $N(E)$ in $B$ is continuous. Using Lemma 1(ii), we deduce that

$$
\text { a. } L, L . a \in N(E) \subset B
$$

for all $a \in A$ and $L \in B(E)$, and that

$$
\left.\begin{array}{rl}
\|a . L\|_{B} \leq C\|a . L\|_{N(E)} \leq C\|a\|_{A}\|L\|_{B(E)}, \\
\|L . a\|_{B} \leq C\|L . a\|_{N(E)} \leq C\|a\|_{A}\|L\|_{B(E)} .
\end{array}\right\}
$$

It follows easily that $B$ is a Banach $A$-bimodule.

Now let $f: A \times A \rightarrow B \subset B(E)$ be a continuous bilinear operator with $\delta^{2} f=0$. By Lemma $6, f=\delta^{1} g$ for some $g: A \rightarrow B(E)$. Hence

$$
g(a b)=a \cdot g(b)-f(a, b)+g(a) . b \quad(a, b \in A) .
$$

It follows from (6) and (7) that $g(a b) \in B$ for all $a, b \in A$, and that

$$
\|g(a b)\|_{B} \leq(\|f\|+2 C\|g\|)\|a\|_{A}\|b\|_{A} .
$$

Consequently the formula

$$
h(\underset{A}{\otimes} b)=g(a b) \quad(a, b \in A)
$$

defines a continuous linear operator $h: A \underset{A}{\otimes} A \rightarrow B$.

It is clear that

$$
h(u)=g(\kappa(u)) \quad(u \in A \underset{A}{\hat{\otimes}} A)
$$

where $\kappa: A \underset{A}{\otimes} A \rightarrow A$ is the operator given by $\kappa(a \otimes b)=a b(a, b \in A)$. Hence $h(\operatorname{ker} \kappa)=0$. By Lemma 3, $\kappa$ is an epimorphism. It follows that there exists a continuous linear operator $g_{1}: A \rightarrow B$ such that $h=g_{1}{ }^{\circ} K$. It is obvious that $g_{1}(a)=g(a)$ for all $a \in A$ and that $f=\delta^{1} g_{1}$. Consequently, $H^{2}(A, B)=0$. The theorem is proved.

TheOREM 6. Let $E$ be a Banach space, and set $N=N(E)$. Then

(i) $H^{2}(N, N)=0$;

(ii) $H^{3}(N, N)=0$ if and only if $E$ has $A P$. 
Proof. The equality (i) follows from Theorem 5. To prove (ii), consider the short complex of $N$-bimodules

$$
0 \rightarrow N \stackrel{i}{\rightarrow} N_{+} \stackrel{a}{\rightarrow} \mathbb{C} \rightarrow 0,
$$

where $\mathbb{C}=N_{+} / N, i$ is the natural embedding of $N$ in $N_{+}$, and $\sigma$ is the natural projection. According to [1, Corollary III.4.1], the complex (8) defines the exact sequence of cohomology groups

$$
\ldots \rightarrow H^{2}\left(N, N_{+}\right) \rightarrow H^{2}(N, \mathbb{C}) \stackrel{\beta}{\rightarrow} H^{3}(N, N) \rightarrow \ldots
$$

Suppose that $E$ does not have $A P$. By Theorem $5, H^{2}\left(N, N_{+}\right)=0$. But then $\beta$ is an embedding, while the group being mapped is nontrivial, as shown in Theorem 4(i). Hence $H^{3}(N, N) \neq 0$.

On the other hand, if $E$ has $A P$, the $H^{3}(N, N)=0$, by Corollary 1 . This completes the proof of (ii).

Finally, we note that, in the case where $E$ is a Hilbert space, the triviality of the groups $H^{2}(N(E), N(E))$ and $H^{3}(N(E), N(E))$ was proved earlier in [10] by B. E. Johnson.

\section{REFERENCES}

1. A. Ya. Helemskii, The homology of Banach and topological algebras (Kluwer, Dordrecht, 1989). (Russian original 1986).

2. A Grothendieck, Produits tensoriels topologiques et espaces nucléaires, Mem. Amer. Math. Soc., 16, 1955.

3. P. Enflo, A counterexample to the approximation problem, Acta Math., 130 (1973), $309-317$.

4. J. Lindenstrauss and L. Tzafriri, Classical Banach spaces, Springer-Verlag, 1977.

5. A. Ya. Helemskii, Banach and polynormed algebras: general theory, representations, homology, Nauka, Moscow, 1989 (Russian). To appear in English, Oxford Univ. Press, 1992.

6. Yu. V. Selivanov, Biprojective Banach algebras, Izv. Akad. Nauk SSSR ser. matem., 43 (1979), 1159-1174 (Russian); Math. USSR-Izv., 15 (1980), 387-399.

7. M. Grosser, Bidualraüme und Vervollständigungen von Banachmoduln, Lecture Notes in Math., 717, Springer-Verlag, 1979.

8. Yu. V. Selivanov, Biprojective Banach algebras and their structure, cohomology, and relation with nuclear operators, Funkc. anal. i pril., 10 (1976), 89-90 (Russian); Funct. Analysis Appl., 10 (1976).

9. Sh. I. Kaliman and Yu. V. Selivanov, On the cohomology of operator algebras, Vest. Mosk. Univ. ser. mat. meh., 29 (5) (1974), 24-27 (Russian); Moscow Univ. Math. Bull., 29 (1974).

10 B. E. Johnson, Perturbations of Banach algebras, Proc. London Math. Soc., 34 (1977), $439-458$.

Chair of Higher Mathematics

Moscow Aircraft Technological Institute N.A.

TsIOLKOVSKY

Petrovka 27, Moscow K-31

103767, Russia. 